\title{
Applicability of Vaisburd and Evdokimov Model to Ionic Targets
}

\author{
F. Sabrin, M. A. Uddin* and A. K. F. Haque \\ Department of Physics, University of Rajshahi, Rajshahi-6205, Bangladesh
}

Received 20 November 2011, accepted in final revised form 20 February 2012

\begin{abstract}
The Vaisburd and Evdokimov proposed an empirical model to calculate the electron impact single ionization cross-sections of atoms and molecules. The model has been applied to some atoms and molecules. To examine the efficiency of the model, the present work applies the model to calculate cross-sections for Ne-isonuclear series $\mathrm{Ne}^{+}, \mathrm{Ne}^{2+}, \mathrm{Ne}^{3+}, \mathrm{Ne}^{4+}$, $\mathrm{Ne}^{5+}, \mathrm{Ne}^{6+}, \mathrm{Ne}^{7+}, \mathrm{Ne}^{8+}, \mathrm{Ne}^{9+}$. The separate sets of values of the parameters of the model are determined by comparison with the available experimental data using a non-linear leastsquares fitting computer code.
\end{abstract}

Keywords: Electron impact ionization; Empirical model; Ionic targets.

(c) 2012 JSR Publications. ISSN: 2070-0237 (Print); 2070-0245 (Online). All rights reserved.

doi: $\underline{\text { http://dx.doi.org/10.3329/jsr.v4i2.8704 }}$ J. Sci. Res. 4 (2), 307-313 (2012)

\section{Introduction}

Electron impact ionization has fundamental importance in understanding structure of atoms, dynamics of collision processes, electron-target interaction, etc. Besides these, the electron impact ionization cross-sections are widely used in such applied areas as mass spectrometry, space physics, radiation science, fusion plasmas, semiconductor industry, lasers, etc. There have been attempts to calculate theoretically the ionization cross sections, producing semi-empirical, classical models and semi-classical models and quantum mechanical methods. Each of the models and methods has its own range of validity. Applied fields need simple-to-use models of sufficient accuracy for rapid generation of cross-sections over wide domains. In 1930, Bethe [1] solved this problem by the methods of quantum mechanics. The Bethe formula is quite simple, but it correctly describes the energy-dependence of the total cross section at high incident energies $E>100 I$, where $I$ is the ionization potential. But in the region of maximum for $I<E<10 I$, it deviates significantly from the measurement data.

Attempts of more precise theoretical calculation of the ionization cross section particularly in and around the peak region using analytical expressions are very rare due to

\footnotetext{
* Corresponding author: uddinmda@yahoo.com
} 
proliferation , very often than not, of resonant ionization, the indirect ionization process in many-electron atoms .

Empirical formulae suggested by Lotz [2], Alkhazov [3], Kim and Rudd [4], and Povyshev et al. [5] have received wide applications, but the indirect ionization has not been included in their structure. The Vaisburd and Evdokimov [6], henceforth referred to as VE model, has the special feature in that it includes a special component describing the resonant ionization and joins elegantly with the Bethe formula valid at high-energy domain. The empirical formulae models proposed by Haque et al.[7,8] provided successful description of cross-sections of electron impact inner-shell ionization of atoms for a wide range of atoms covering up to the energy of $\mathrm{GeV}$ order with inclusion of ionic and relativistic ingredients into the structures of the models .

The VE model has been applied to some neutral atoms and molecules and demonstrated a good success in describing the available experimental data. This model involves four main parameters and two parameters connecting it to the Bethe formula. To the best of our knowledge, the model has not been tried on ionic targets. This leaves a prospect for testing the efficacy of the model in the case of ionic targets. Encouraged by its fascinating structure, we have applied the VE model to Ne-isonuclear series $\mathrm{Ne}^{+}$, $\mathrm{Ne}^{2+}, \mathrm{Ne}^{3+}, \mathrm{Ne}^{4+}, \mathrm{Ne}^{5+}, \mathrm{Ne}^{6+}, \mathrm{Ne}^{7+}, \mathrm{Ne}^{8+}, \mathrm{Ne}^{9}$. The application of the model to atomic ions, not explored yet, might be a new facet of the model.

Section 2 gives the outline of the model. Section 3 contains discussion of results with graphs. We give the conclusion in section 4 .

\section{Outline of the VE model}

Bethe considered an inelastic collision of an electron with an atom in the context of quantum mechanics in framework of the first Born approximation and derived a formula for the EISICS. The Born approximation is applicable if the velocity of the incident electron is much greater than the velocities of atomic electrons. As a result, the Bethe theory describes well ionization of the atoms by fast electrons. The Bethe formula can be ived using the Fermi golden rule for the probability of atom transition from the ground to an excited state per unit time [9].

Introducing the variable

$$
u=E / I-1
$$

The Bethe cross section is

$$
\sigma=\frac{4 \pi a_{o}^{\prime} R^{2} N M_{i}^{2}}{I^{2}} \frac{\ln (u+1)}{u+1}
$$

Here $N$ is the number of electrons on the shell to be ionized, $M_{i}^{2}$ is a constant. $R$ is the Rydberg energy $=13.606 \mathrm{eV}$ and $a_{0}$ is the Bohr radius. 
Vaisburd and Evdokimov [6] assume that the interaction of the incident electron with the Fermi electron-hole system of the atom is resonant in character for electron energies $I<E<10 I$. In this region, the VE model represents EISICS by the expression

$$
\sigma_{L}=\frac{\text { const }}{\sqrt{\Gamma^{2}+\left(\frac{u}{\alpha}-\frac{\alpha}{u}\right)^{2}}}
$$

where $\Gamma$ is the parameter determining the curve width in the region of maximum, and $\alpha$ also a parameter specifying the position of this maximum.

Bethe demonstrated that, for high energies of the incident electron, the dipole interaction of the incident electron with the atomic system makes the main contribution to the inelastic scattering cross section and in particular, to the ionization cross section. As a consequence, the Bethe formula describes well the dependence of the total ionization cross section on the energy of the incident electron when it exceeds several tens of ionization potentials. In the region of maximum ionization cross section, when the energy of the incident electron is in the interval $I<E<10 I$, the ionization cross section is resonant in character, as demonstrated above. In the VE model, the following mechanism of the interaction of the incident electron with the atom has been suggested. For small energies, the incident electron is resonantly captured by the atomic system, and forms a common excited system with electrons of the shell. Then the system undergoes the resonant transition from the excited to the ground state with emission of one or two electrons. At high incident energy, such a common system has no time to be formed, and the electron interacts with one of the electrons on the shell according to the Bethe theory.

A comparison with the available experimental data demonstrates that the formula for the total ionization cross section in the vicinity of the maximum must be described by a resonant curve similar to Lorentz distribution Eq. 2.3, and for high energies of the incident electron, it must be transformed into the Bethe formula Eq. 2.2. The VE model is the result of the combination of the formulae in Eqs. 2.3 and 2.2 and is expressed as

$$
\sigma_{L B}=\frac{4 \pi a_{o}^{2} N R^{2} M_{i}^{2}}{I^{2}}\left(\frac{\beta}{\sqrt{\Gamma^{2}+\left(\frac{u}{\alpha}-\frac{\alpha}{u}\right)^{2}}} \frac{1}{\exp \left(\frac{u-u_{F}}{u_{o}}\right)+1}+\frac{\ln (u+1)}{u+1} \frac{1}{\exp \left(\frac{u_{F}-u}{u_{o}}\right)+1}\right)
$$

Here $M, \Gamma, \alpha, \beta, u_{o}, u_{f}$ are the parameters of the model .

\section{Results and Discussion}

Ionization potentials for the atomic ions, considered in this work, are calculated using Dirac-Hartree-Fock code [10] . The values of the parameters in formula (2.4) have been 
obtained for the species, from the available experimental data using the least-squares method. The value of $M_{i}^{2}$ was first calculated by fitting the Bethe cross section Eq. 2.2 to the available experimental data for high energies of the incident electron. The remaining parameters $\Gamma, \alpha, \beta, u_{o}, u_{f}$ entering in the formula 2.4 have been calculated, for the derived value of $M_{i}^{2}$, from the entire body of the experimental data. The parameters of the VE model for the $\mathrm{Ne}$ isonuclear ions $\mathrm{Ne}^{+}, \mathrm{Ne}^{2+}, \mathrm{Ne}^{3+}, \mathrm{Ne}^{4+}, \mathrm{Ne}^{5+}, \mathrm{Ne}^{6+}, \mathrm{Ne}^{7+}, \mathrm{Ne}^{8+}, \mathrm{Ne}^{9+}$ are given in Table 1. Figs. 1 and 2 compare the VE cross-sections with available experimental data.

Table 1. Parameters of the VE model for Ne isonuclear series.

\begin{tabular}{cccccccccc}
\hline Ions & $I(\mathrm{eV})$ & Orbital & $N$ & $M_{i}^{2}$ & $\Gamma$ & $\alpha$ & $\beta$ & $u_{\mathrm{o}}$ & $U_{\mathrm{F}}$ \\
\hline $\mathrm{Ne}^{+}$ & 41.1 & $2 p^{5}$ & 5 & 0.455 & 38.61 & 0.084 & 15.6 & 0.39 & 0.1150 \\
$\mathrm{Ne}^{2+}$ & 63.5 & $2 p^{4}$ & 4 & 0.546 & 43.43 & 0.112 & 24.3 & 2.29 & 4.1303 \\
$\mathrm{Ne}^{3+}$ & 92.5 & $2 p^{3}$ & 3 & 1.188 & 16.12 & 17.99 & 9.18 & 4.72 & 1.3998 \\
$\mathrm{Ne}^{4+}$ & 126.2 & $2 p^{2}$ & 2 & 1.274 & 38.69 & 17.35 & 15.4 & 0.33 & 2.1364 \\
$\mathrm{Ne}^{5+}$ & 157.9 & $2 p^{1}$ & 1 & 1.901 & 7.442 & 19.42 & 0.60 & 0.01 & 0.0638 \\
$\mathrm{Ne}^{6+}$ & 207.5 & $1 s^{2} 2 s^{2}$ & 4 & 0.605 & 17.69 & 0.206 & 0.51 & 156.501 & 0.0004 \\
$\mathrm{Ne}^{7+}\left(1^{\text {st }}\right)$ & 239.1 & $1 s^{2} 2 s^{1}$ & 3 & 0.689 & 0.014 & 7.693 & 1.93 & 0.12 & 1.7509 \\
$\mathrm{Ne}^{7+}\left(2^{\text {nd }}\right)$ & & & & 0.712 & 46.55 & 0.027 & 20.1 & 2.41 & 2.3749 \\
$\mathrm{Ne}^{8+}$ & 1196 & $1 s^{2}$ & 2 & 0.723 & 17.06 & 6.757 & 4.66 & 0.03 & 1.1991 \\
$\mathrm{Ne}^{9+}$ & 1360.6 & $1 s^{1}$ & 1 & 0.472 & 6.611 & 7.939 & 8.69 & 3.03 & 0.0002 \\
\hline
\end{tabular}

The sources of experimental data are Dolder et al.[11], Muller et al. [12], Donets and Ovsyannikov [13], Diserens et al. [14], Blaha and Davis [15] for $\mathrm{Ne}^{+}$; Donets and Ovsyannikov [13], Danjo et al. [16], Bannister [17] for $\mathrm{Ne}^{2+}$; Gregory et al.[18], Donets and Ovsyannikov [13] for $\mathrm{Ne}^{3+}$, Bannister [17], Duponchelle et al.[19] for $\mathrm{Ne}^{4+}$, Donets and Ovsyannikov [13], Bannister [16] and Duponchelle et al.[19] for $\mathrm{Ne}^{5+}$, Donets and Ovsyannikov [13], Bannister [17] and Duponchelle et al.[19] for $\mathrm{Ne}^{6+}$, Donets and Ovsyannikov [13], Defrance et al. [20] and Duponchelle et al.[19] for $\mathrm{Ne}^{7+}$, Donets and Ovsyannikov [13] and Duponchelle et al.[19] for $\mathrm{Ne}^{8+}$, Donets and Ovsyannikov [13] for $\mathrm{Ne}^{9+}$.

As apparent from Figs. 1 and 2, the VE model produces a good level of agreement with one or another set of experimental data for the all the members of the Ne isonuclear series, even successfully reproducing folds in the data. 

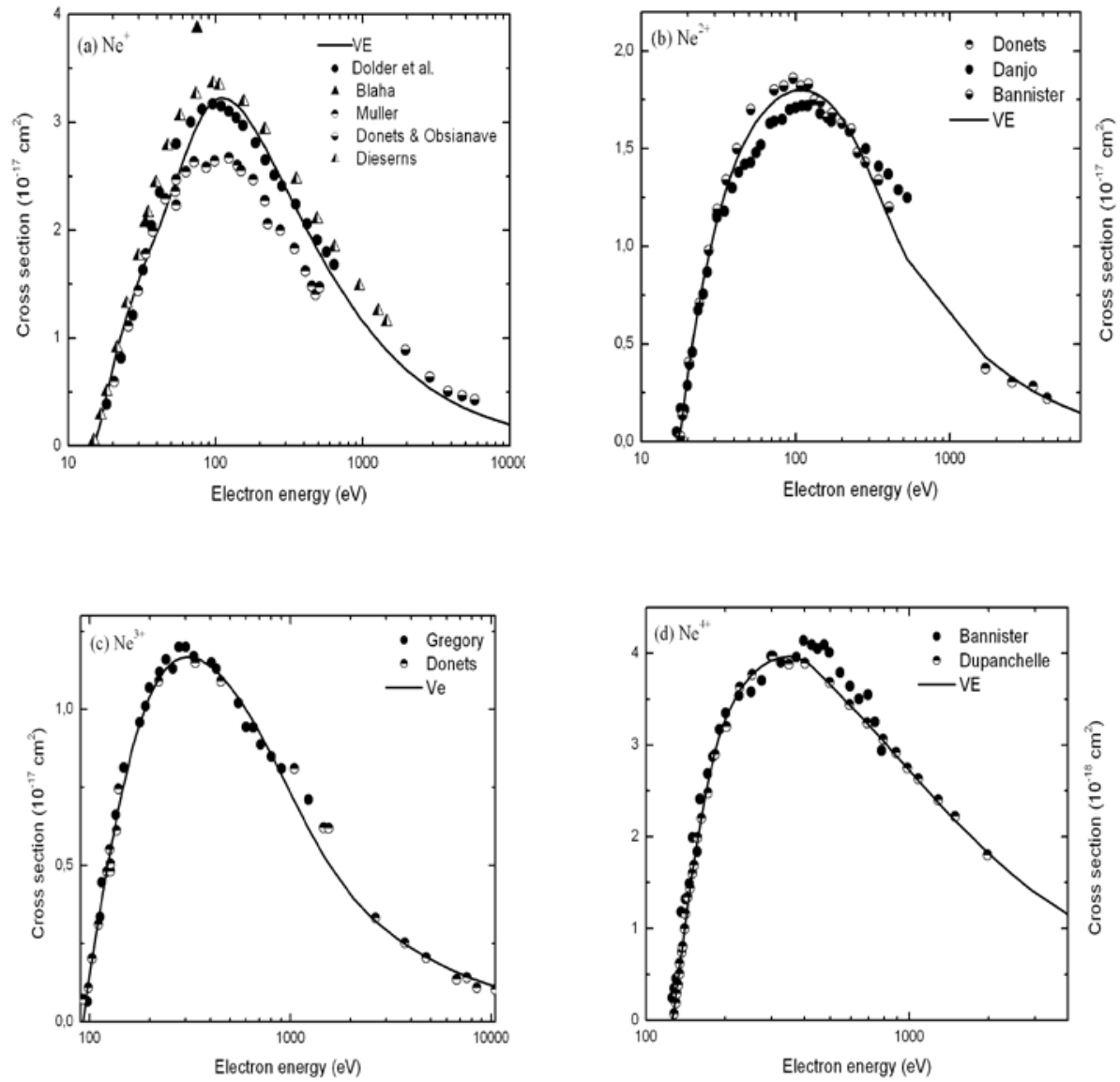

Fig. 1. Electron impact ionization of Ne-isonuclear series, (a) $\mathrm{Ne}^{+}$, (b) $\mathrm{Ne}^{2+}$, (c) $\mathrm{Ne}^{3+}$, and (d) $\mathrm{Ne}^{4+}$,
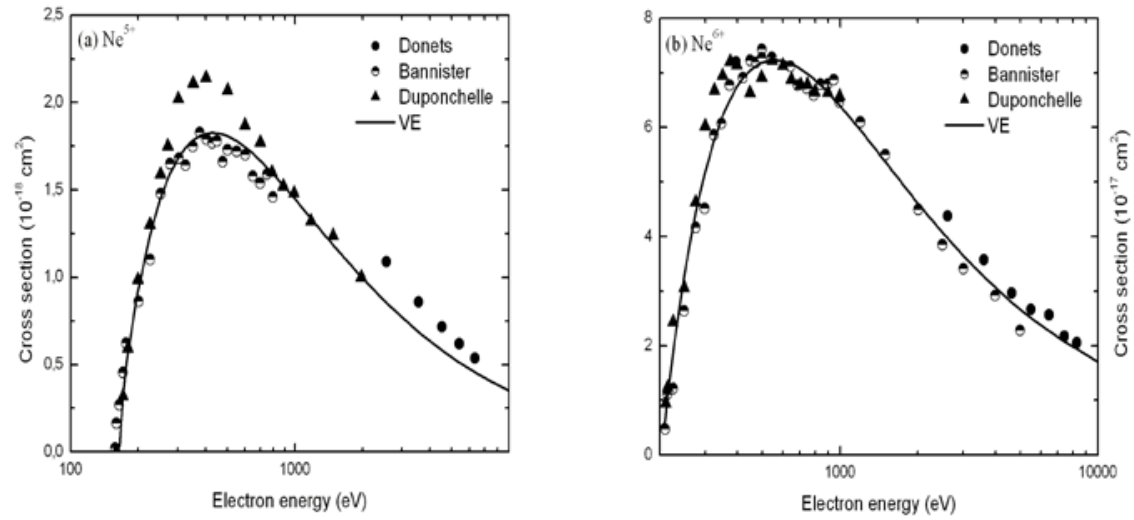

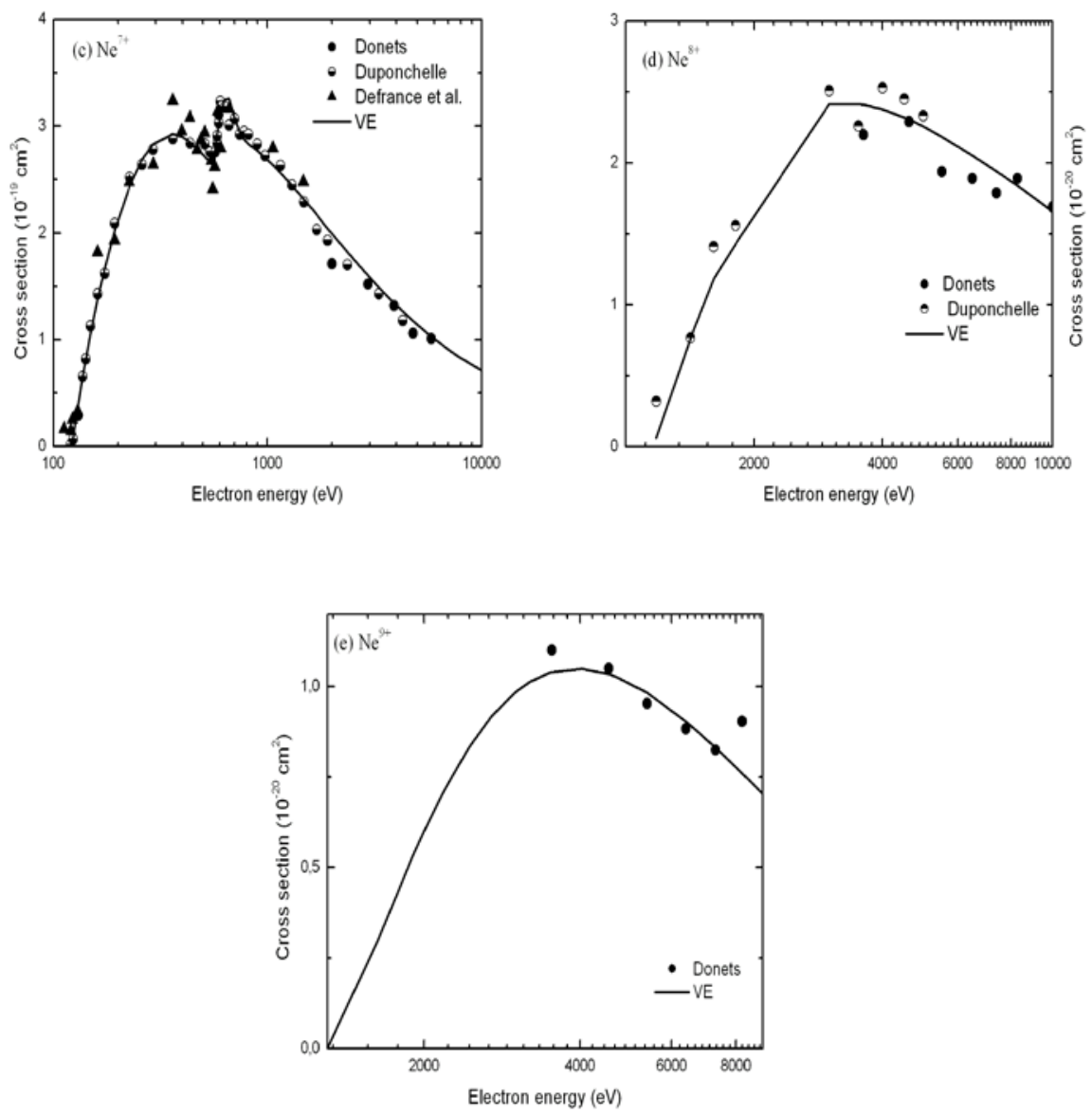

Fig. 2.. Electron impact ionization of Ne-isonuclear series, (a) $\mathrm{Ne}^{\mathrm{J+}}$, (b) $\mathrm{Ne}^{\mathrm{ot}}$, (c) $\mathrm{Ne}^{7+}$, (d) $\mathrm{Ne}^{8+}$ and (e) $\mathrm{Ne}^{9+}$.

\section{Conclusion}

In this work the, we have found that in the region of maximum for $I<E<10 I$, the EISICS is described by a resonant curve similar to Lorentz distribution. We conclude that the resonance excitation and decay of the Fermi electron-hole system makes the main contribution to the ionization by a slow electron. The resonant formula in conjunction with the smoothly connected Bethe formula forms a lucrative method for the calculation of the EISICS for ionic targets. This study supplements the result of the earlier VE calculations for neural atomic and molecular targets for a wide range of energies, thereby widening its scope of applicability to ionic targets.

This study demonstrates that the VE model with optimum values of the parameters describes successfully the experimental data for ionic targets. Using the optimized values of the parameters, the VE model may be applied to calculate the EISICSs at any arbitrary 
energy for the aforesaid targets studied in this work. This study bears testimony to the efficacy of the model for the smoothing of scarce and scattered experimental data and theoretical cross-sections, which is required for modeling in applied sciences. This model appears to be a promising method for fast and accurate generation of cross-sections even when indirect process of ionization is involved.

\section{References}

1. H. Bethe, Ann. Phys. (Lipzig) 5, 325 (1930). http://dx.doi.org/10.1002/andp.19303970303

2. W. Lotz, Z. Phys. 232, 101 (1970). http://dx.doi.org/10.1007/BF01393132

3. G. D. Alkhazov, Zh. Thkh. Fiz. 40, 97 (1970).

4. Y. -K. Kim and M. E. Rudd, Phys. Rev. A 50, 3954 (1994). http://dx.doi.org/10.1103/PhysRevA.50.3954

5. V. M. Povyshev, A. A. Sadovoy, V. P. Shevelko, G. D. Shirkov, E. G. Vasina, and V. V. Vatulin, JINR-E9-2001-148, [online]. Available: http://www1.jinr.ru/Preprints/2001/e9-2001148.pdf

6. D. I. Vaisburd and K. E. Evdokimov, Russian Phys Journal 46 (11), 1160 (2003). http://dx.doi.org/10.1023/B:RUPJ.0000024368.93924.a7

7. A. K. F. Haque, M. Shahjahan, M. R. Talukder, M. Shahjahan, M. A. Uddin, A. K. Basak, and B. C. Saha, J. Phys. B: At. Mol. Opt. Phys. 43, 115201 (2010). http://dx.doi.org/10.1088/0953-4075/43/11/115201

8. A. K. F. Haque, M. A. Uddin, M. Shahjahan, M. R. Talukder, A. K. Basak, and B. C. Saha, Advances in Quantum Chemistry, Volume 6 (Academic Press, USA, 2011).

9. L. D. Landau and E. M. Lifshits, Quantum Mechanics [In Russian], Nauka, Moscow (1974).

10. M. Y. Amusia and L. V. Chernysheva, Computations of Atomic Processes (IOP Publishing, Bristol, 1997). http://dx.doi.org/10.1887/0750302291

11. K. T. Dolder, M. F. A. Harrison, and P. C. Thonemann, Proc. Roy. Soc. (London) A 274, 546 (1963).

12. A. Muller, E. Salzborn, R. Frodl, R. Beeker, H. Klein, and H. Winter, J. Phy. B: At. Mol. Phys. B 13, 1877 (1980). http://dx.doi.org/10.1088/0022-3700/13/9/018

13. E. D. Donets and V. P. Ovsyannikov, Sov. Phys. -JETP 53, $466(1981)$.

14. M. J. Diserens,M. F. A. Harrison, and A. C. H. Smith, J. Phys. B 17, L621 (1984). http://dx.doi.org/10.1088/0022-3700/17/18/003

15. M. Blaha and J. Davis, J. Naval Research Lab. Memo. 4245, (1980) (unpublished).

16. A. Danjo. A. Matsumoto, S. Ohtani, H. Suzuki, H. Tawara, K.. Wakiya, and M. Yoshino, J. Phys. Soc. Japan 53, 4091 (1984). http://dx.doi.org/10.1143/JPSJ.53.4091

17. M. E. Bannister, Phys. Rev. A 54, 1435 (1996). http://dx.doi.org/10.1103/PhysRevA.54.1435

18. D. N. Gregory, D. F. Dittner, and D. H. Crandall, Phys. Rev. A 27, 724 (1982). http://dx.doi.org/10.1103/PhysRevA.27.724

19. M. Duponchelle, M. Khoulid, E. M. Onalim, H. Zhany, and P. J. Defrance, J. Phys. B 30, 729 (1997). http://dx.doi.org/10.1088/0953-4075/30/3/025 